Meta

Journal des traducteurs

Translators' Journal

\title{
L'enseignement de la traduction médicale : un double défi ?
}

\section{Hannelore Lee-Jahnke}

Volume 46, numéro 1, mars 2001

Traduction médicale et documentation / Medical translation and documentation

URI : https://id.erudit.org/iderudit/003445ar

DOI : https://doi.org/10.7202/003445ar

Aller au sommaire du numéro

Éditeur(s)

Les Presses de l'Université de Montréal

ISSN

0026-0452 (imprimé)

1492-1421 (numérique)

Découvrir la revue

Citer cet article

Lee-Jahnke, H. (2001). L'enseignement de la traduction médicale : un double défi ? Meta, 46(1), 145-153. https://doi.org/10.7202/003445ar

\section{Résumé de l'article}

Quel est l'intérêt particulier que présente la traduction médicale en situation d'enseignement universitaire ? Qu'est-ce qui a changé dans ce domaine au fil du temps ? De quels facteurs nouveaux doit tenir compte le professeur en prodiguant cette discipline ? Voilà quelques questions auxquelles nous tentons de répondre en nous basant sur notre propre expérience en situation d'enseignement universitaire. 


\title{
L'enseignement de la traduction médicale: un double défi?
}

\author{
HANNELORE LEE-JAHNKE \\ Université de Genève, Genève, Suisse
}

\begin{abstract}
RÉSUMÉ
Quel est l'intérêt particulier que présente la traduction médicale en situation d'enseignement universitaire? Qu'est-ce qui a changé dans ce domaine au fil du temps? De quels facteurs nouveaux doit tenir compte le professeur en prodiguant cette discipline? Voilà quelques questions auxquelles nous tentons de répondre en nous basant sur notre propre expérience en situation d'enseignement universitaire.
\end{abstract}

\section{ABSTRACT}

What is the relevance of medical translation in a university-level course? What has changed in this field over the years? What new elements should be taken into account when teaching the course? These are some of the questions that we shall attempt to answer from our own experience in university teaching.

\section{MOTS-CLÉS/KEYWORDS}

traduction médicale, enseignement de la traduction médicale, textes scientifiques, terminologie médicale, phraséologie médicale

\section{Introduction}

Nombreuses sont les études qui ont été consacrées à l'enseignement de la traduction et à la problématique inhérente à cette discipline (Delisle 1997). Les spécificités des langues de spécialités et notamment celles du langage médical ont fait et font également de plus en plus l'objet d'ouvrages de référence. Il suffit dans ce contexte de penser au numéro spécial de la présente revue de 1986 qui s’intitulait «Traduction et terminologie médicales", mais aussi à une des dernières parutions de l'American Translators Association: Translation and Medicine (1998).

\section{L'intérêt de la traduction médicale}

De nos jours, l'intérêt est double: d'une part historique car, avec Henri Van Hoof (1993: 1-2), nous pouvons dire: «Avec la traduction religieuse, la traduction médicale est probablement une des branches les plus anciennes de l'activité traduisante: les souffrances de l'âme et du corps ont toujours été au centre des préoccupations de l'homme. Le plus ancien des documents serait le Corpus Hippocraticum, une compilation des enseignements d'Hippocrate faite au $\mathrm{II}^{\mathrm{e}}$ siècle avant notre ère par des médecins grecs d'Alexandrie.»

D'autre part, la traduction médicale prend actuellement une ampleur inespérée sur le marché et cela malgré la position de l'anglais comme lingua franca, un fait maintes fois confirmé par des enquêtes effectuées au sein des associations professionnelles de traducteurs. Former des spécialistes dans ce domaine semble donc suffisamment justifié.

Meta, XLVI, 1, 2001 


\section{L'enseignement de la traduction médicale au niveau universitaire}

\subsection{Le moment propice dans le cursus}

Si un plan d'étude prévoit la discipline qui nous intéresse ici, l'établissement du cursus doit forcément tenir compte du stade du savoir des étudiants, car il faut bien entendu qu'ils soient familiarisés avec le maniement des textes spécialisés. Dans un cursus de quatre ans, il s'est avéré judicieux de placer la traduction médicale autant que les autres traductions spécialisées - dans les deux dernières années. Ainsi, les connaissances préalables à ce type de traduction sont censées être acquises, par exemple les théories applicables aux textes scientifiques ${ }^{1}$, les différentes typologies de textes ${ }^{2}$, ainsi que l'importance qu'on accorde chaque fois au public cible.

\subsection{La motivation}

Comme tout enseignement aux adultes exige de la motivation, une première tâche que doit accomplir le professeur est de susciter cette motivation pour une matière de prime abord - difficile. Comment? D'une part, cela présuppose de bien connaître le marché, avec tous ses points forts, ses nouveautés aussi - et il y en a. D'autre part, les particularités des couples de langues sont à expliciter.

Cela suppose, par ailleurs, de bien connaître le groupe d'étudiants. Il faut se demander - et la question a déjà été posée à différentes reprises, notamment par Gile (1986:26) — qui sont les meilleurs traducteurs médicaux: les traducteurs avec des connaissances en médecine ou bien les médecins avec des connaissances traductologiques? En ce qui concerne la Suisse et l'Allemagne, on peut dire que la tendance va plutôt vers le traducteur avec des connaissances en langage médical ${ }^{3}$.

La motivation des étudiants s'accroît également grâce à une meilleure connaissance - pour ne pas dire approfondie - de la matière. Pour y parvenir, une solide introduction aux méandres du langage médical avec toutes ses difficultés est indispensable. Pour ce faire, nous disposons d'excellents ouvrages, faits par des spécialistes, qui ne facilitent pas seulement l'apprentissage en classe mais qui permettent également un travail individuel à l'apprenant ${ }^{4}$.

Nous avons également pu constater que la motivation est augmentée, et de beaucoup, par un enseignement interdisciplinaire que la faculté de médecine de l'Université de Genève nous accorde parfois. Les étudiants en traduction se retrouvent avec les étudiants en médecine dans des petits groupes de travail qui relèvent du tutorial. Une participation active aux questions-réponses s'avère des plus utiles pour les uns et pour les autres.

\subsection{Connaissances traductologiques fondamentales}

Les apprentis traducteurs doivent se familiariser avec les théories applicables aux textes scientifiques. Nous pensons ici surtout à la typologie des textes de Susanne Göpferich (1995) et de Rosemarie Gläser (1998) qui permettent une certaine catégorisation et ainsi de trouver facilement le registre de langue approprié.

En ce qui concerne le contenu, il s'agit de distinguer l'information non définie d'un point de vue socioculturel, qui vaut pour toutes les langues, de l'information définie exclusivement pour certains groupes culturels. Il est indispensable, dès le début, 
de préciser les objectifs d'un tel enseignement, de mettre en relief les difficultés inhérentes à cette langue de spécialité et d'employer des modèles d'enseignement qui lient théorie et pratique.

Ces cours visent nécessairement un triple but:

a) connaître la structure des textes dans les langues impliquées,

b) maîtriser la langue spécialisée,

c) s'approprier les connaissances d'au moins un domaine.

En ce qui concerne l'allemand, comme d'autres langues d'ailleurs, la terminologie est fortement imprégnée par des termes d'origine grecque ou latine. Toutefois, il s'agit, par ailleurs, d'attirer l'attention des étudiants au fait qu'un certain nombre de notions «germanisées» sont devenues des termes techniques. En outre, avec l'évolution de l'anglais comme lingua franca, nombre de mots anglais ont trouvé leur place dans la langue de spécialité, notamment en ce qui concerne les domaines de pointe, tels que l'imagerie par résonance magnétique.

Du point de vue de la traduction, il convient d'attirer l'attention des étudiants sur le fait que texte source et texte cible peuvent varier considérablement en longueur. Cela est vrai notamment dans le couple de langues anglais-allemand ${ }^{5}$.

En outre, l'étudiant doit développer une vigilance particulière à l'égard des faux amis qui le guettent, surtout dans le domaine des affixes.

\section{Les difficultés}

Tout pédagogue et psychologue sait qu'on ne maitrise la peur qu'en se familiarisant avec ce qui nous afflige. Il est donc primordial, dès le départ, d'attirer l'attention des étudiants sur un certain nombre de difficultés de la traduction médicale, difficultés auxquelles nous avons en partie déjà fait allusion.

\subsection{La terminologie}

Dans bien des cas, le grec et le latin régissent encore le langage médical. C'est un fait historique bien établi. C'est là en général où les étudiants en traduction, qui très souvent ne maîtrisent plus ces langues, prennent peur. L'approche développée par Maurice Rouleau (1994), c'est-à-dire de diviser ces mots et d'analyser leur contenu, est des plus utiles: elle permet de familiariser petit à petit l'apprenti traducteur avec la matière et de le mettre en confiance. Ce genre d'exercice doit être effectué à répétition et certainement chaque fois qu'un domaine nouveau est abordé.

Puisque la traduction médicale est basée sur des connaissances cognitives spécifiques que normalement seul le spécialiste maîtrise et que son objectif premier est l'information, nous devons être vigilants face aux domaines qui se chevauchent et qui rendent la compréhension difficile, un point déjà traité en 1990 par Amal Jammal.

La condition sine qua non à cela est l'usage des textes parallèles, de la documentation sous toutes ses formes et des nouveaux outils à notre disposition. Nous $y$ reviendrons plus loin. 


\subsection{Les acronymes}

Peu de domaines sont autant truffés d'acronymes que les textes médicaux. Bien entendu, il y a dans ce domaine des ouvrages de référence qui sont utiles ${ }^{6}$, mis à part les dictionnaires spécialisés. Cependant, comme nous rencontrons non seulement des acronymes standardisés mais aussi des acronymes spécifiques aux auteurs, il s'agit d'abord de les distinguer et, surtout, d'en avertir l'étudiant.

Les acronymes spécifiques aux auteurs devraient être facilement décelables si les auteurs en question suivent le schéma IMRAD ${ }^{7}$, utilisé surtout aux États-Unis mais qu'on trouve de plus en plus dans la presse médicale européenne. Dans ce contexte, l'auteur est tenu d'expliquer l'acronyme qu'il a créé pour ses besoins. Il reste alors la tâche ardue pour le traducteur de trouver ou même de créer un acronyme. Notre collaboration avec la faculté de médecine a montré que les auteurs sont souvent très coopératifs s'ils maîtrisent la langue cible. Dans le cas contraire, ils insistent dans bien des cas pour garder leur «trouvaille».

\subsection{Les éponymes}

D’après la typologie établie par Van Hoof (1993), il y a une différence selon qu'un nom est banalisé ou qu'il est resté intact. Il est intéressant d'y voir la triple distinction que fait l'auteur:

a) éponymes identiques dans les textes source et cible,

b) éponymes différents dans les textes source et cible,

c) manque d'éponyme dans une des deux langues.

Cette problématique exige souvent de longues recherches et des discussions avec les experts. Là encore, l'enseignement interdisciplinaire était d'une grande utilité.

\subsection{La dominance de l'anglais}

Nous avons fait allusion plus haut à la terminologie anglaise qui a trouvé son entrée dans bien des langues. Question de facilité? Très souvent. Facteur temps? Aussi. Des mots tels que compliance ou spin font partie intégrante du vocabulaire médical en allemand comme en français. Pour s'en convaincre, il suffit de consulter le glossaire sur l'imagerie par résonance magnétique ${ }^{8}$ auquel nous faisions allusion plus haut.

\subsection{La phraséologie médicale}

Les difficultés de la phraséologie dans ce domaine sont, dans la plupart des cas, inhérentes à des facteurs extralinguistiques, c'est-à-dire le genre de texte. Il suffit pour cela de présenter des rapports médicaux rédigés par des médecins de différents pays. Dans le même contexte, il faut rendre attentif les étudiants à une certaine redondance spécifique au domaine médical. Bien entendu, la traduction peut être moins redondante, mais il s'agit d'être véritablement familiarisé avec la matière pour «s'ingérer» de beaucoup dans le texte de départ. N'oublions pas que les textes spécialisés en médecine consistent en une description et classification de phénomènes concrets : objectifs, processus analytiques, méthodes, causes et effets. 


\section{Qu'est-ce qui a changé au fil du temps?}

\subsection{Les outils de documentation}

L'importance de la documentation dans l'enseignement de la traduction a pris de plus en plus d'ampleur, notamment dans un domaine comme celui qui nous intéresse ici, où très souvent la documentation livrera la solution au problème de traduction. Il s'agit de répondre aux questions quand, comment et quel outil de documentation présenter.

La documentation devrait, selon les pédagogues de renommée, faire partie intégrante du cursus universitaire (Delisle 1997). De nos jours, il y a certes à ajouter l'usage des nouvelles technologies (Lee-Jahnke 1998a: 119), mais tel n'est pas encore le cas partout. Prenons un exemple de situation «idéale» d'enseignement. Vu la masse d'information, il est indispensable d'enseigner aux étudiants comment choisir et classer de façon systématique les documents.

Un tel cours devrait idéalement se situer tout au début des études afin de faciliter dès le départ l'apprentissage systématique avant même de faire savoir aux étudiants qu'il y a environ 10 millions d'articles ${ }^{9}$ qui remplissent les bibliothèques de médecine ou que, tous les mois, sont publiées dans le monde environ 4000 revues médicales.

Cependant, comme l'objectif de l'enseignement n'est pas de faire peur mais plutôt de rassurer et de trouver des solutions, il convient d'ajouter à ces chiffres qu'environ 10 à $15 \%$ des publications seulement dans le domaine médical ont une valeur scientifique durable. Afin de maîtriser cette masse d'information, nous avons eu recours à la lecture en trois phases décrite par David Jewell (dans Jones et Kinmonth 1995). De quoi s'agit-il?

a) browsing: feuilleter rapidement un livre pour déceler ce qui nous intéresse;

b) reading for information: être à la recherche, dans la littérature, de solutions à des problèmes spécifiques;

c) reading for research: effectuer une lecture ciblée pour acquérir des connaissances approfondies.

Le seul survol de la littérature n'exige pas un ordre particulier mais, par contre, si notre objectif est une recherche précise, un manque de méthode entraîne une grande perte de temps qu'aucun futur traducteur ne peut se permettre.

La formation doit donc fournir des outils adéquats pour répondre à ces exigences. C'est ainsi que, même avant l'ère informatique, il y avait un document de grande valeur appelé Excerpta Medica; on y trouvait tous les résumés de la littérature spécialisée et parfois même les traductions. La recherche prenait pourtant encore du temps.

Aujourd'hui, nous avons les banques de données et notamment DataStar ${ }^{10}$, une banque qui offre surtout des informations médicales et pharmaceutiques. Ce qui est intéressant pour le traducteur, c'est que cette banque couvre tout dans le domaine médical depuis la recherche jusqu'à la vente ${ }^{11}$.

Dans notre enseignement, nous soulignons surtout Medline, une banque qui contient environ un tiers des 10 millions d'articles auxquels nous avons fait allusion plus haut. Elle a l'avantage d'être interrogeable de pratiquement toutes les bibliothèques du monde occidental ${ }^{12}$. Cette banque de données est alimentée par la National Library of Medicine aux États-Unis et contient plus de 3800 revues spécialisées d'environ 70 pays. Les informations peuvent être obtenues sous trois formes différentes: 
a) sur papier (Index Medicus, mis à jour annuellement, existe également sous forme électronique),

b) en ligne par Internet,

c) sur cédérom (la banque de données complète comporte de 10 à 18 disques selon l'éditeur).

L'utilisation de cet outil extrêmement précieux pour le traducteur spécialisé en médecine qu'est Medline passe par la connaissance de son fonctionnement. L'étudiant devrait d'abord savoir qu'un article peut être recherché:

a) par mots-clés dans le titre, par le nom de l'auteur ou l'institut où l'étude a été effectuée;

b) à l'aide d'un thésaurus de titres médicaux: le MeSH (Medical Subject Heading).

Les étudiants apprécient les exemples pratiques de recherche. Pour cela, il faut savoir un certain nombre d'abréviations telles que:

$\begin{array}{lll}\mathrm{ab} & = & \text { résumé (abstract) } \\ \mathrm{au} & = & \text { auteur (author) } \\ \mathrm{jn} & = & \text { revue (journal) } \\ \mathrm{me} & = & \text { terme du MeSH }(\text { MeSH-term) } \\ \mathrm{ti} & = & \text { mot dans le titre (word in title) } \\ \mathrm{tw} & = & \text { mot dans le texte (word in text) } \\ \mathrm{ui} & = & \text { identificateur unique (unique identifier) } \\ \mathrm{yr} & = & \text { année de parution (year of publication) }\end{array}$

Admettons qu'on cherche un article qui concerne le suivi (follow up) des patients et qui a paru dans le British Medical Journal; il s'agit de faire la recherche suivante:

a) taper follow up.ti, ce qui donne probablement des centaines de titres;

b) taper British Medical Journal.jn, ce qui donne à nouveau de nombreux articles;

c) combiner a) et b), ce qui va aboutir à l'article recherché.

Un autre cas de figure peut être que vous voulez familiariser vos étudiants à un certain domaine médical et donc recherchez de la documentation. Disons que ce soit sur le diabète. Il va d'abord falloir insérer la notion, c'est-à-dire diabetes mellitus, sans spécifier le domaine. Puis on vous demandera si vous voulez tous les articles ou seulement certains exemples spécifiques. La banque fournit alors tous les sous-titres. Le même résultat peut s'obtenir tout simplement en tapant ceci :

*diabetes mellitus/

L'astérisque indique le mot-clé de l'article et la barre, l'expression du MeSH.

Parfois, la recherche ne donne rien ou presque. Cela peut vouloir dire que la banque ne contient pas d'article pertinent mais, ce qui est encore plus probable, c'est que la recherche a été menée d'une façon trop exclusive en se référant aux sous-titres. Il s'agit donc de toujours indiquer aux apprenants de chercher d'après les mots-clés dans le texte. Voyons l'exemple suivant: vous cherchez de la documentation à propos du rôle de l'Aspirine dans le traitement de l'infarctus du myocarde. Vous avez alors les possibilités suivantes:

\section{- Myocardial infarction/pc ou Myocardial infarction/dt et aspirin/tu}

Cette recherche vous donnera tous les articles de Medline qui traitent de l'emploi de l'Aspirine dans le traitement de l'infarctus du myocarde. Cela peut, de nos jours, renvoyer à plus de 200 articles, fait qui ne résout pas forcément les problèmes liés à la 
documentation trop abondante que Wilss (1996) a déploré à plusieurs reprises. Le moyen de cibler davantage la recherche serait le suivant:

- Myocardial infarction/and aspirin/

- limit 1 to human

- limit 2 to AIM journals

- limit 3 to review articles

Au début de l'an 2000, cette façon de rechercher donnait environ 40 articles.

Par contre, si le résultat obtenu n'est pas celui espéré, il se peut qu'il y ait eu une erreur lors de l'introduction de l'article dans Medline. En outre, il ne faut pas négliger le fait qu'il y ait des domaines pour lesquels Medline n'est pas en mesure de fournir toute la documentation. C'est notamment le cas de la psychologie et de certains domaines extra-cliniques de la pharmacologie. Là encore, le professeur peut suggérer des banques de données médicales qui traitent de façon ciblée de certains sujets. Par exemple:

- Aidsline regroupe tous les travaux sur le SIDA depuis 1980;

- Allied and Alternative Medicine présente les travaux sur l'acupuncture et l'homéopathie ;

- Cancer-CD est composé de Cancerlit et remonte jusqu'en 1984. La version sur cédérom est mise à jour chaque trimestre.

Il y a aussi des banques de données thématiques d’ordre plus général:

- EmBase est une banque de données d'Excerpta Medica qui contient des travaux sur des médicaments et sur la pharmacologie en général ainsi que sur les domaines biomédicaux spécialisés. Cette banque recouvre davantage le marché européen et est bien souvent plus à jour que Medline. La version sur cédérom est mise à jour tous les mois.

- Current Contents Search contient des sommaires de revues déjà parues ou même à paraître. Depuis 1990, cette banque de données bénéficie d'une mise à jour hebdomadaire.

- Cochrane Library ${ }^{13}$ est une banque de données non dépourvue d'intérêt pour ses résumés et dont la mise à jour est semestrielle.

Autant que possible, les modèles d'apprentissage devraient permettre, selon les textes et sujets à traiter, d'utiliser les banques de données appropriées et de se familiariser avec elles afin d'apprendre à en faire un choix judicieux.

\subsection{L'attitude du futur traducteur face à la documentation}

Il est primordial d'initier les étudiants à une lecture critique des textes parallèles afin qu'ils puissent se documenter de façon efficace. Parfois, une remise en question de l'usage d'une certaine notion par l'auteur du texte est indispensable. En d'autres termes, un bon traducteur ne peut se contenter d'une seule source, d'un seul texte parallèle. Cette démarche est extrêmement importante dans l'enseignement et peut judicieusement être divisée en documentation intra- et extra-textuelle.

La première source est, bien entendu, le texte à traduire, car le texte de départ, s'il n'est pas clair à un endroit, peut fournir une explication à un autre endroit. Cette recherche intra-textuelle permet, dans bien des cas, une recherche bien plus ciblée par la suite. Dans certains cas, elle sera même «auto-suffisante». 
En ce qui concerne la documentation extra-textuelle, nous pensons à ce que nous avons dit plus haut mais également aux ouvrages médicaux de référence, qui existent en plusieurs langues et permettent des lectures parallèles extrêmement précieuses pour l'apprenant. Parmi ces ouvrages, retenons celui d'Harrison ${ }^{14}$, qui existe en anglais, en allemand et en français, ou celui de Ganong ${ }^{15}$, qu'on peut consulter en anglais, en allemand, en français et en italien.

Le plurilinguisme en Suisse a donné lieu à un Compendium suisse des médicaments en allemand et en français. Ce compendium a été longtemps réservé aux seuls médecins. Actuellement, il existe une version destinée à un large public qu'on peut trouver dans les bibliothèques universitaires de même que sur le Web ${ }^{16}$. Il est évident qu'Internet offre de nos jours une source précieuse pour la recherche documentaire. En annexe, nous avons inclus quelques adresses qui nous semblent utiles.

L'enseignement de la traduction médicale ne peut pas ignorer que les banques de données, elles aussi, ne sont pas toujours dépourvues d'erreurs. La critique y est donc autant de mise que pour les documents sur papier.

Une autre mise en garde à l'apprenant est de se demander lesquels des documents ou banques de données méritent d'être consultés. Selon quelles caractéristiques fautil faire le tri? Voici quelques points qui nous ont été utiles en enseignement:

a) consulter les articles d'abord et de préférence en langue d'origine (ce qui n'est pas toujours facile en médecine);

b) vérifier le degré de fiabilité du document en fonction des trois points suivants:

1. l'auteur, ce qui est déterminant pour le niveau de langue,

2. le lieu de publication,

3. la date de publication.

Tous ceux qui sont familiers avec la terminologie savent bien sûr faire ces démarches et les feront de façon automatique.

En résumé, il est impératif:

1. de savoir comment chercher le plus efficacement un article, soit par les mots dans le texte (titre, résumé ou les deux) ou par les suffixes des domaines (auteur, revue, année de parution).

2. d'effectuer la recherche sous MeSH.

3. de bien cibler le sujet afin d'exclure le matériel inutile.

4. de parcourir les résumés les plus récents.

5. de cerner le but exact d'une étude afin de savoir si elle est utile pour la traduction.

6. d'établir la terminologie d'usage s'il s'agit d'un document d'un laboratoire pharmaceutique.

Les outils modernes ont non seulement modifié le travail des traducteurs mais ont aussi marqué un tournant dans l'enseignement de la traduction, notamment dans celui de la traduction médicale. Il permettent de travailler de façon plus efficace et avec une qualité accrue.

L'enseignement de la traduction médicale est donc bel et bien un double défi pour le professeur: celui de l'enseignement de la traduction selon les règles de la didactique inhérente à cette discipline, et celui de la maîtrise d'une matière fascinante par voie interdisciplinaire et au moyen d'outils modernes et constamment mis à jour. 


\section{NOTES}

1. La typologie des textes de Susanne Göpferich permet d'abord une identification exacte du genre de texte et donne ensuite la possibilité de bien choisir le registre de langue.

2. Voir aussi le schéma sur la traduction scientifique de Rosemarie Gläser (1998).

3. Nous avons examiné cette problématique dans Lee-Jahnke (1998b).

4. Nous pensons surtout à l'ouvrage de Maurice Rouleau.

5. Voir également l'exemple que nous avons donné dans l'article précité

6. Nous pensons notamment dans le domaine germanophone au livre d'Ursula Spranger (1990).

7. IMRAD $=$ Introduction, Materials or Methods, Results and Discussion.

8. Voir dans Eurospin Quarterly ou bien dans le $\mathrm{n}^{\circ} 13$ de Parallèles.

9. Voir aussi le vol. 315 du British Medical Journal (19 juillet 1997).

10. Depuis 1981. Adresse Web: <http://dsweb.krinfo.ch/test>

11. À titre d'exemple: Aidsline, Allied and Alternative Medicine, American Medical Association Journals, BioBusiness, Cancerlit, Current Contents Search, Forensic Science, Health News Daily, Immunoclones, The Lancet, Medical Toxicology and Health, Meditec, Medline.

12. Il faut avoir accès à Datastar sous Windows 3.1 (ou plus récent) avec au moins 16 Mo de mémoire vive.

13. Cette banque porte le nom de l'épidémiologue Archie Cochrane qui, en 1972, trouvait important de constituer un registre central pour les essais cliniques.

14. L'original anglais s'intitule Principles of Internal Medecine.

15. L'original anglais s'intitule Physiology.

16. Ce compendium est aussi accessible sur le Web: <http://www.kompendium.ch/app/search_f.cfm $>$.

\section{RÉFÉRENCES}

Delisle, J. (1997): La traduction raisonnée, Ottawa, Presses de l'Université d'Ottawa.

Ganong, W. F. (1974) : Physiologie, Berlin, Springer Verlag.

GiLE, D. (1986) : «La traduction médicale doit-elle être réservée aux seuls traducteurs médecins?», Meta, 31-1, p. 26-31.

GLÄSER, R. (1998) : “Fachsprachen und Funktionalstile. Art. 16”, HSK-Fachsprachen, pp. 199-208.

GöPfERICH, S. (1995): "A Pragmatic Classification of LSP Texts in Science and Technology," Target, 7-2, pp. 305-326.

Greenhalgh, T. (1997): "How to Read a Paper," British Medical Journal, 315.

Harrison, T. R. (1987): Principles of Internal Medicine, 11th ed., New York and Montreal, McGraw-Hill.

Jammal, A. (1990) : «L'étude des langues des spécialités médicales», Meta, 35-1, p. 50-54.

Jones, R. and A.-L. Kinmonth (1995) : Critical reading for primary care, Oxford, Oxford University Press.

Lee-JahnKe, H. (1998) : «La traduction médicale: un défi?», Traduire, 1.

Lee-Jahnke, H. (1998) : “Training in Medical Translation,” ATA Monograph X, pp. 81-93.

PARAllèles (1991): Atelier de traduction sur la résonance magnétique nucléaire, Genève, ETI, $\mathrm{n}^{\circ} 13$.

Rouleau, M. (1994) : La traduction médicale, Brossard, Linguatech.

Schefe, P. (1981): Zur Funktionalität der Wissenschaftssprache - Am Beispiel der Medizinischen Wissenschaftssprache, München, Theo Bungarten, pp. 356-371.

Spranger, U. (1990): Abkürzungen in der Medizin und ihren Randgebieten, Stuttgart, Fischer Verlag.

Schweizerisches Arzneimittelkompendium (1996), Basel, Documed.

VAN Hoof, H. (1993): «Histoire de la traduction médicale en Occident», Cahiers de l'Institut linguistique de Louvain, 19-1/2.

Wilss, W. (1996) : Übersetzungsunterricht, Tübingen, Gunter Narr.

YLÖNEN, S. (1993) : "Stilwandel in wissenschaftlichen Artikeln der Medizin. Zur Entwicklung der Textsorte "Originalarbeiten" in der Deutschen Medizinischen Wochenschrift 1884-1989", Fachtextpragmatik. Forum für Fachsprachenforschung (H. Schröder, ed.), Tübingen, Gunter Narr, pp. 81-98. 